\title{
Experimentos Numéricos para o Gradiente do FWI via Método dos Estados Adjuntos: uma comparação entre o domínio do tempo e o domínio da frequência.
}

\author{
Isadora A. S. de Macedo (UFPA, Faculdade de Geofísica, Belém (PA), Brasil \& INCT-GP), Natiê Almeida Albano (UFPA, \\ Faculdade de Geofísica, Belém (PA))
}

\section{Introdução}

Um dos desafios dos métodos sísmicos é gerar uma imagem em alta resolução da subperfície através do desenvolvimento de complexos sistemas de aquisição. Uma das formas de investigar as estruturas da subsperfície é através da construção da matriz das derivadas de Fréchet, que indica a sensibilidade dos dados observados aos parâmetros do meio. A utilização da matriz de sensibilidade se tornou inviável em sistemas de aquisição complexos por conta do aumento do custo computacional, o que possibilitou o avanço de novos métodos de imageamento. Claerbout (1971) mostrou que um refletor existe onde os campos de onda da fonte e do receptor coincidem no tempo e no espaço. A refletividade como um atributo da amplitude dos traços em um ponto do refletor forneceu uma imagem migrada possível de ser interpretada estratigraficamente.

Lailly (1983) e Tarantola (1994) reformularam o princípio proposto por Claerbout (1971) como um problema de otimização, onde deseja-se minimizar o erro entre o dado observado e o dado calculado a partir de uma modelagem numérica. O gradiente da função objetivo (soma dos quadrados dos resíduos entre o campo calculado e observado) é obtido através da correlação cruzada entre o campo de onda propagado pela fonte e o campo residual propagado a partir dos receptores. O modelo de perturbação obtido, quando adicionado ao modelo de velocidade inicial, dá origem a um modelo de velocidade atualizado que, por sua vez, é usado como modelo inicial na próxima iteração para minimizar a função objetivo. Essa estimativa é realizada para todas as fontes, receptores e para o tempo total de aquisição, logo, é computacionalmente expensiva.

Com o advento de aquisições sísmicas multi-azimute e de longos offsets e de fontes com maior banda de frequência, a inversão do campo completo de onda - Full Waveform Inversion (FWI) - ganhou destaque com os trabalhos pioneiros de Pratt (1990) e Mora (1987).

Com a FWI é possível estimar os parâmetros físicos da subsuperfície com alta precisão e resolução. Esta abordagem por consistir em um método de inversão não linear pode levar o problema a uma solução que não consiste em um mínimo global, surgindo a necessidade de adotar estratégias que contornem o problema provocado pela não-linearidade da FWI, como a utilização de um modelo de velocidade inicial próximo do desejado, a presença de informações de baixa frequência nos dados observados e conhecimento da fonte utilizada na aquisição dos dados. A FWI pode ser implementada tanto no domínio do tempo quanto no domínio da frequência. A utilização de uma ou outra abordagem depende do tipo do problema. No domínio da frequência, a questão da não-linearidade pode ser contornada com a inversão multi escala, que consiste em inverter o dado para uma única frequência, ou para uma banda de frequência separadamente.

A FWI iterativamente atualiza o modelo de parâmetros com uma quantidade que depende do gradiente da função objetivo. De modo geral, a computação do gradiente no domínio do tempo está dividida em três partes, a 1) modelagem direta no tempo dos campos de onda a partir da fonte, 2) modelagem do campo de onda adjunto reverso no tempo a partir das fontes adjuntas, localizadas na posição dos receptores, e 3) aplicação de um operador de condição de imagem. O cálculo do gradiente é o principal fator a influenciar o alto custo computacional da FWI, uma vez que requer a correlação cruzada entre o campo da fonte e o campo adjunto para todos os instantes de tempo, para todos os receptores e para todas as fontes.

Dentro deste contexto, este trabalho visa descrever as etapas realizadas para a implementação computacional do gradiente da função objetivo para a equação acústica da onda no domínio do tempo, além de apresentar os seus experimentos numéricos e compará-los com o gradiente da função objetivo obtido a partir da formulação no domínio da frequência.

\section{Formulação matemática: método dos estados} adjuntos

\section{Domínio do tempo}

Seja a equação acústica da onda com densidade constante dada por:

$$
\frac{\partial^{2} p}{\partial t^{2}}-v^{2}(\mathbf{x}) \nabla^{2} p=s(t) \delta\left(\mathbf{x}-\mathbf{x}_{\mathbf{s}}\right),
$$

onde $p(\mathbf{x}, t)$ é o campo de pressão, $v(\mathbf{x})$ é a velocidade do meio, $s(t) \delta\left(\mathbf{x}-\mathbf{x}_{\mathbf{s}}\right)$ o termo fonte e fazendo $c(\mathbf{x})=v^{2}(\mathbf{x})$ a equação de estado para o problema inverso será dada por:

$$
\frac{\partial^{2} p}{\partial t^{2}}-c(\mathbf{x}) \nabla^{2} p-s(t) \delta\left(\mathbf{x}-\mathbf{x}_{\mathbf{s}}\right)=0 .
$$

Define-se a função objetivo como:

$$
J=\frac{1}{2} \sum_{s} \sum_{r} \sum_{t}\left[p^{o b s}\left(t, \mathbf{x}_{\mathbf{r}} ; \mathbf{x}_{\mathbf{s}}\right)-p\left(t, \mathbf{x}_{\mathbf{r}} ; \mathbf{x}_{\mathbf{s}}\right)\right]^{2},
$$


onde $p^{o b s}\left(t, \mathbf{x}_{\mathbf{r}} ; \mathbf{x}_{\mathbf{S}}\right)$ e $p\left(t, \mathbf{x}_{\mathbf{r}} ; \mathbf{x}_{\mathbf{S}}\right)$ representam o campo observado e calculado, respectivamente.

De posse da função objetivo e da equação de estado, pode-se construir a lagrangeana:

$$
\begin{array}{r}
\mathscr{L}(p, \Lambda \mid c)=\frac{1}{2} \sum_{s} \sum_{r} \sum_{t}\left[p^{o b s}\left(t, \mathbf{x}_{\mathbf{r}} ; \mathbf{x}_{\mathbf{S}}\right)-p\left(t, \mathbf{x}_{\mathbf{r}} ; \mathbf{x}_{\mathbf{s}}\right)\right]^{2} \\
+\sum_{s} \int_{\Omega} d x \int_{0}^{t} d t \Lambda\left[\frac{\partial^{2} p\left(t, \mathbf{x}_{\mathbf{r}} ; \mathbf{x}_{\mathbf{S}}\right)}{\partial t^{2}}-c(\mathbf{x}) \nabla^{2} p\left(t, \mathbf{x}_{\mathbf{r}} ; \mathbf{x}_{\mathbf{S}}\right)-s(t) \delta\left(\mathbf{x}-\mathbf{x}_{\mathbf{s}}\right)\right]
\end{array}
$$

O termo $\frac{\partial \mathbf{u}}{\partial m}$ constitui a matriz de sensibilidade. Como calculá-la explicitamente é caro computacionalmente, (4t))iza-se o método dos estados adjuntos para computar o gradiente da função objetivo sem a necessidade de calcular todas as componentes dessa matriz. Para tanto, deriva-se a equação 8 em relação aos parâmetros:

$$
\frac{\partial \mathbf{u}}{\partial m}=-L^{-1} \frac{\partial L}{\partial m} \mathbf{u}
$$

Substituindo a equação 11 em 10, tem-se:

cuja fonte adjunta é o resíduo do campo observado e calculado. Uma vez calculado o campo adjunto $\Lambda$ pela equação de estados adjuntos (5) e aplicando as condições de fronteira impostas à $\Lambda$ que exige que a mesmo seja nula nas bordas, a variação do funcional - gradiente da função objetivo - em relação às propriedades físicas será dada por:

$$
\delta \mathscr{L}=\sum_{s} \int_{\Omega} d x \int_{0}^{T} d t\left[\delta c(\mathbf{x}) \nabla^{2} p\left(t, \mathbf{x}_{\mathbf{r}} ; \mathbf{x}_{\mathbf{S}}\right) \Lambda\right]
$$

\section{Domínio da frequência}

Esta seção mostra, de forma geral, como obter o gradiente da função objetivo no domínio da frequência. Aspectos da modelagem no domínio da frequência são comentados nas próximas seções. Seja a equação da onda no domínio da frequência definida por:

$$
-\omega^{2} M \mathbf{u}(\omega)+K \mathbf{u}(\omega)=f(\omega),
$$

onde $M$ representa a matriz de massa (mass matrix), $K$ representa a matriz de rigidez (stiffness matrix), $\omega$ é a frequência angular, $f$ é a fonte e u é o campo de onda discretizado. A equação 7 pode ser expressa como um sistema linear da forma:

$$
L \mathbf{u}=\mathbf{f}
$$

onde os termos $\left(-\omega^{2} M+K\right)$ da equação 7 compõe a matriz $L$

Define-se a função objetivo como:

$$
\chi(m)=\chi[\mathbf{u}(m)]
$$

$$
\frac{\partial \chi}{\partial m}=-\mathbf{u} \frac{\partial L^{T}}{\partial m}\left(L^{-1}\right)^{T} \nabla_{u} \chi
$$

Definindo-se:

$$
L^{H} \cdot \mathbf{u}^{\dagger}=-\nabla_{u} \chi
$$

e substituindo em 12, tem-se:

$$
\frac{\partial \chi}{\partial m}=\mathbf{u} \frac{\partial L^{H}}{\partial m} \mathbf{u}^{\dagger}
$$

A equação 14 é o gradiente da função objetivo no domínio da frequência. Os índices $H$ e $†$ representam o transposto hermitiano para a matriz e para o vetor, respectivamente. O campo u é obtido através da modelagem direta no domínio da frequência e o campo adjunto $\mathbf{u}^{\dagger}$ é obtido através da solução da equação 13 , que também constitui uma modelagem, porém com a fonte sendo o resíduo entre os campos. Essas modelagens, por sua vez, são alcançadas através da solução do sistema linear mostrado na equação 8.

\section{Experimentos numéricos: par fonte receptor}

Esta seção mostra os resultados gerados com o algoritmo que calcula o gradiente para um par fonte-receptor. A equação 6 foi a que fora implementada neste trabalho para gerar o gradiente: é realizada a correlação cruzada entre o campo adjunto (modelado a partir da equação 5) e o laplaciano do campo de onda direto. Utilizou-se a equação 1 parametrizada para $c(\mathbf{x})=v^{2}(\mathbf{x})$ para computar o campos de onda direto para o modelo calculado. O procedimento para gerar estes gradientes, para todos os modelos testados, foi o seguinte:

1. Define-se a posição da fonte, a posição do receptor e a frequência pico do pulso fonte. 
Tabela 1: Parâmetros do modelo homogêneo

\begin{tabular}{lll}
\hline \multicolumn{3}{c}{ Parâmetros do modelo homogêneo } \\
\hline $\mathrm{nx}$ & Número de pontos na direção x & 301 \\
$\mathrm{nz}$ & Número de pontos na direção z & 301 \\
$\mathrm{dx}$ & Incremento espacial na direção x & $10.0 \mathrm{~m}$ \\
$\mathrm{dz}$ & Incremento espacial na direção z & $10.0 \mathrm{~m}$ \\
$f$ & Frequência pico do pulso fonte & $10 \mathrm{~Hz}$ \\
$t$ & Tempo total de modelagem & $4 \mathrm{~s}$ \\
$\mathrm{Vc}$ & Velocidade do modelo calculado & $3500 \mathrm{~m} / \mathrm{s}$ \\
$\mathrm{Vo}$ & Velocidade do modelo observado & $3000 \mathrm{~m} / \mathrm{s}$
\end{tabular}

2. Define-se um modelo de velocidade observado e registra-se o traço sísmico na posição do receptor.

3. Define-se um modelo de velocidade inicial (calculado) e registra-se o traço na posição do receptor e armazena-se o laplaciano do campo de onda direta propagado na modelagem.

4. Calcula-se o resíduo entre os traços registrados com o modelo observado e com o modelo calculado.

5. Utilizando o resíduo como fonte, propaga-se, reversamente no tempo, o campo de onda adjunto a partir da posição do receptor e armazena-se os campos retropropagados.

6. Para o mesmo ponto no espaço e no tempo, calculase a correlação cruzada entre o laplaciano do campo direto e o campo adjunto que gerará um ponto imagem no gradiente.

\section{Modelo homogêneo}

O primeiro resultado diz respeito ao gradiente calculado em um meio homogêneo cujos parâmetros estão descritos na tabela ??. A figura 2 mostra o gradiente calculado para duas frequências distintas: $5 \mathrm{~Hz}$ e $10 \mathrm{~Hz}$.

O teste no modelo homogêneo mostra o efeito isolado das ondas transmitidas no gradiente do FWI (Transmission $F W I$ ), uma vez que no modelo homogêneo não haverá ondas refletidas. $O$ destaque se dá para as banana kernels. Com o offset fixo, mantendo a mesma escala e mudando apenas a frequência, é possível notar o efeito da mesma no gradiente: as frequências mais baixas mostram ter uma contribuição maior no gradiente quando comparada às frequências mais altas.

\section{Modelo com refletor}

Um teste no modelo com um refletor foi realizado para validar o algoritmo do gradiente e para verificar a relação existente entre o offset e a profundidade de iluminação do gradiente. Neste caso, foram realizados dois modelos com profundidades distintas do refletor: $1000 \mathrm{~m}$ e $2500 \mathrm{~m}$. Os parâmetros dos modelos estão na tabela 2.

[1-2] [3-10]

Primeiramente, nos dois modelos de refletor usados para teste, os modelos observado e calculado possuem a mesma profundidade do refletor, alterando apenas a velocidade da camada inferior, o que faz com que o efeito da onda direta seja eliminado e os eventos de reflexão sejam isolados, fazendo com que as rabbit ears kernels

\begin{tabular}{lll}
\hline \multicolumn{3}{c}{ Parâmetros do modelo homogêneo } \\
\hline $\mathrm{nx}$ & Número de pontos na direção x & 301 \\
$\mathrm{nz}$ & Número de pontos na direção z & 301 \\
$\mathrm{dx}$ & Incremento espacial na direção x & $10.0 \mathrm{~m}$ \\
$\mathrm{dz}$ & Incremento espacial na direção z & $10.0 \mathrm{~m}$ \\
$f$ & Frequência pico do pulso fonte & $10 \mathrm{~Hz}$ \\
$t$ & Tempo total de modelagem & $4 \mathrm{~s}$ \\
$\mathrm{Vc}$ & Velocidade do modelo calculado & $3500 \mathrm{~m} / \mathrm{s}$ \\
Vo & Velocidade do modelo observado & $3000 \mathrm{~m} / \mathrm{s}$ \\
\multicolumn{3}{c}{ Figura 1: Parâmetros do modelo homogêneo }
\end{tabular}

(a)

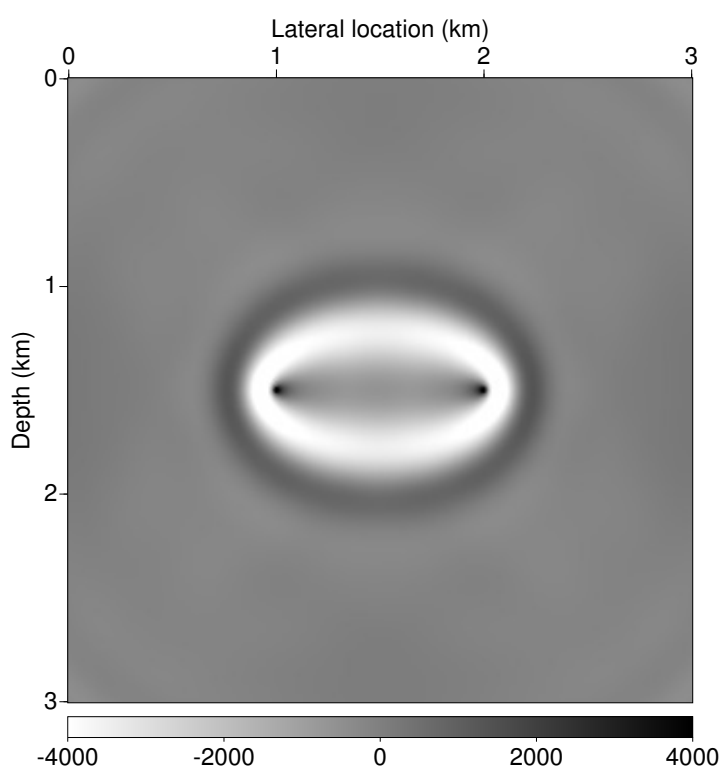

(b)

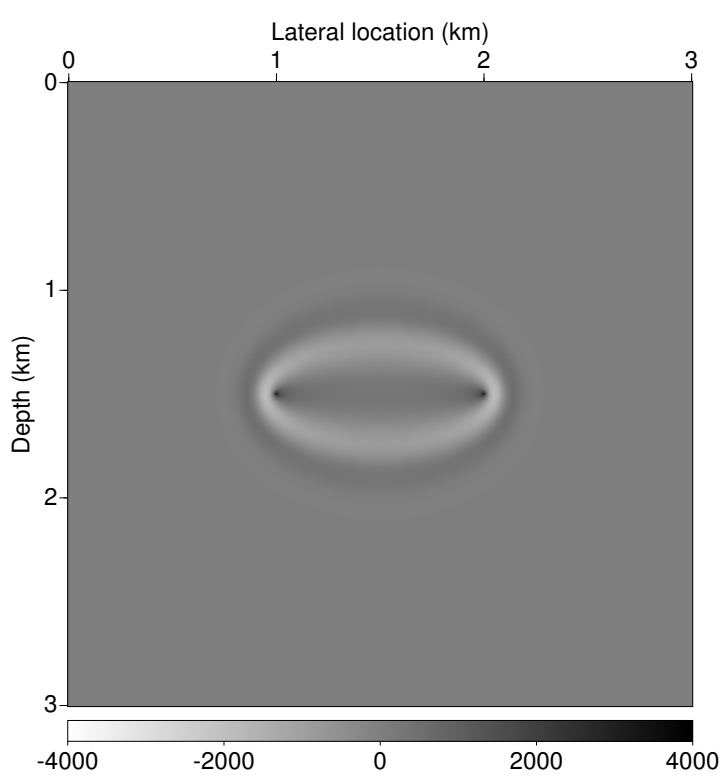

Figura 2: Gradientes calculados para o meio homogêneo com diferentes frequências pico do pulso fonte. a) Frequência de $5 \mathrm{~Hz}$. b) Frequência de $10 \mathrm{~Hz}$. 
(a)
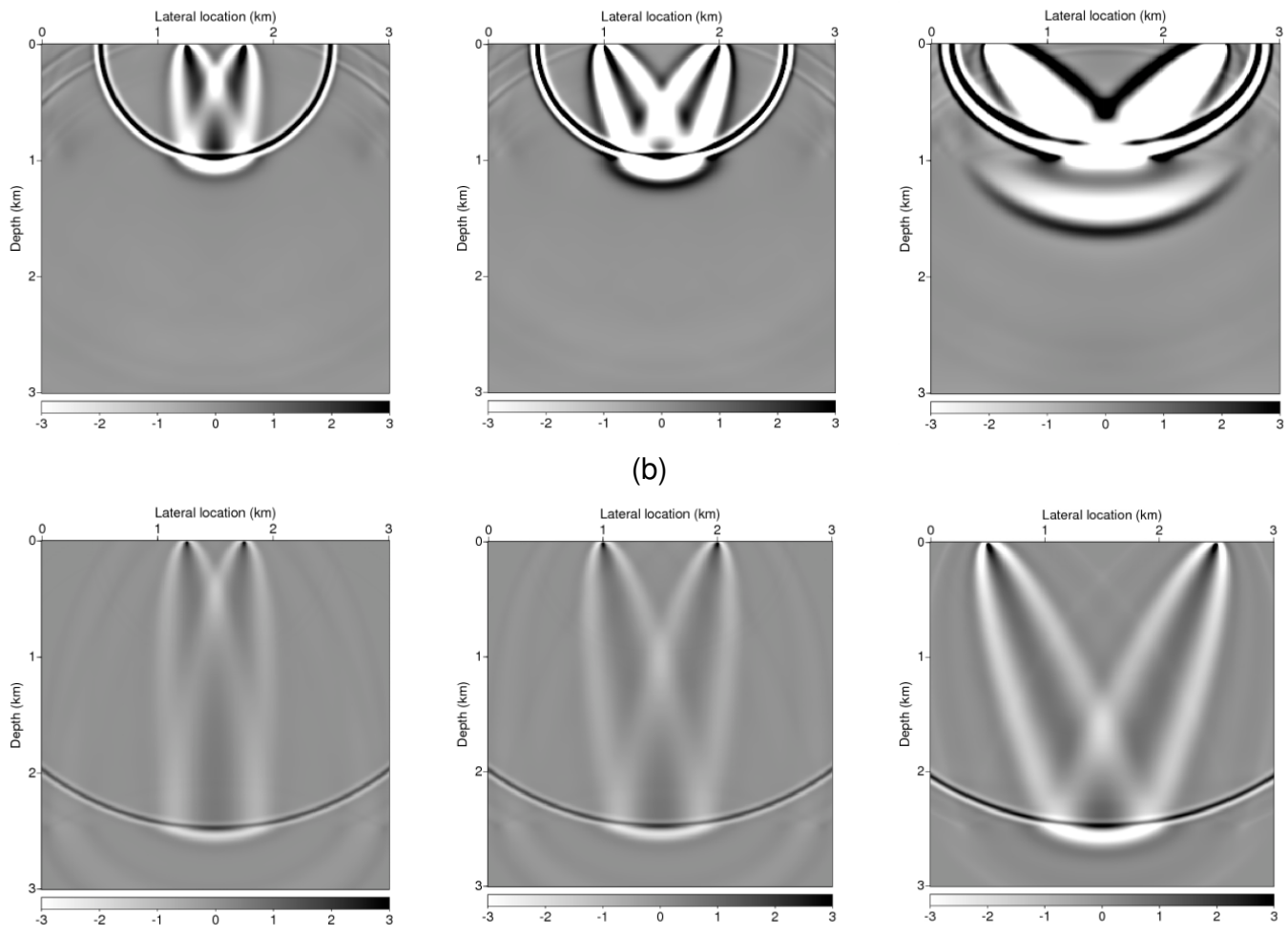

Figura 5: Gradiente para o offset de 500m para um refletor em diferentes profundidades. a) Refletor a 1000m. b) Refletor a $2500 \mathrm{~m}$.

Tabela 2: Parâmetros do modelo com refletor

\begin{tabular}{lll}
\hline \multicolumn{3}{c}{ Parâmetros do modelo com refletor } \\
\hline $\mathrm{nx}$ & Número de pontos na direção x & 301 \\
$\mathrm{nz}$ & Número de pontos na direção z & 301 \\
$\mathrm{dx}$ & Incremento espacial na direção x & $10.0 \mathrm{~m}$ \\
$\mathrm{dz}$ & Incremento espacial na direção z & $10.0 \mathrm{~m}$ \\
$f$ & Frequência pico do pulso fonte & $10 \mathrm{~Hz}$ \\
$t$ & Tempo total de modelagem & $4 \mathrm{~s}$ \\
$\mathrm{Vc}$ & Velocidade inferior do modelo calculado & $3500 \mathrm{~m} / \mathrm{s}$ \\
$\mathrm{Vo}$ & Velocidade inferior do modelo observado & $3000 \mathrm{~m} / \mathrm{s}$ \\
$\mathrm{V}$ & Velocidade da camada superior & $1500 \mathrm{~m} / \mathrm{s}$
\end{tabular}

sejam destacadas, o que constitui o Reflection FWI. O teste mostrou a influência do offset na iluminação do refletor no gradiente, o que consequentemente afeta a recuperação da propriedade física do refletor na inversão. Ao comparar o gradiente do offset de $500 \mathrm{~m}$ (figura 5) com o gradiente do offset de $2000 m$ (figura ??), fica claro que o aumento do offset possibilita a iluminação de refletores nas porções mais profundas do modelo e que quando o refletor está raso e o offset é longo, as porções mais rasas atingem o seu máximo de iluminação. Ao relacionar o resultado deste teste com o resultado dos testes realizados no modelo homogêneo, que mostrou que a resposta do gradiente é melhor e mais nítida para as menores frequências, pode-se concluir que é preciso que haja um balanço entre a frequência utilizada no FWI e o offset para que seja possível obter o melhor resultado no gradiente e se possa iluminar até as regiões mais profundas do modelo, uma vez que as baixas frequências e os longos offsets produzem a melhor iluminação.
Tabela 3: Parâmetros da aquisição: modelo Marmousi

\begin{tabular}{lll}
\hline \multicolumn{2}{c}{ Parâmetros da aquisição: modelo Marmousi } \\
\hline $\mathrm{N}_{s h o t}$ & Número de tiros & 138 \\
$\mathrm{~N}_{r c v}$ & Número de receptores & 180 \\
$\mathrm{~d}_{s h o t}$ & Espaçamento entre os tiros & $65 \mathrm{~m}$ \\
$\mathrm{~d}_{r c v}$ & Espaçamento entre os receptores & $50 \mathrm{~m}$ \\
$t$ & Tempo total de modelagem & $4 \mathrm{~s}$ \\
$\mathrm{dt}$ & Tempo de registro do traço & $0.004 \mathrm{~s}$ \\
\hline
\end{tabular}

\section{Experimentos numéricos: várias fontes e receptores e comparação com o domínio da frequência}

\section{Modelo Marmousi}

Ao simular uma aquisição completa no modelo Marmousi e calcular o gradiente, comparamos os resultados obtidos no domínio do tempo com os resultados obtidos a partir de um algoritmo que calcula o gradiente no domínio da frequência. Os parâmetros da aquisição neste modelo estão indicados na tabela 3 e os modelos observado e calculado estão na figura 6.

O teste no modelo Marmousi mostrou que o gradiente para a aquisição completa se assemelha ao modelo. A parte mais rasa do gradiente ficou com as amplitudes maiores, o que é natural, já que com a profundidade os campos de onda perdem amplitude devido a divergência esférica, espalhamento geométrico, dentre outros fatores. É possível que as faixas de maior amplitude no topo da imagem sejam devido a linha de receptores posicionados a uma profundidade de 50 metros e à profundidade da 
(a)

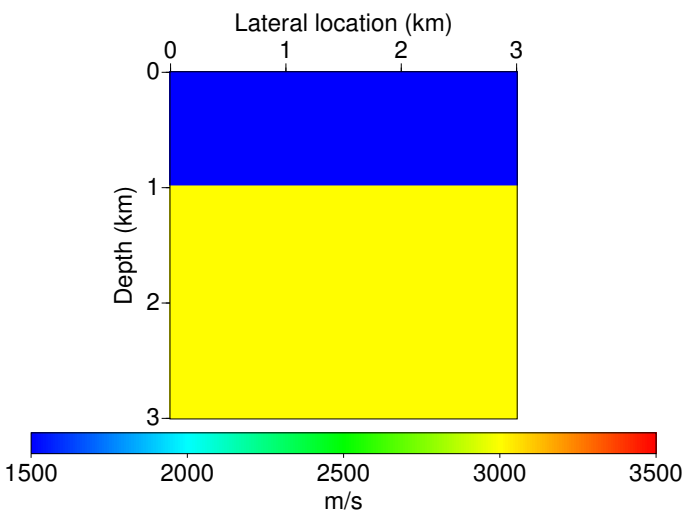

(b)

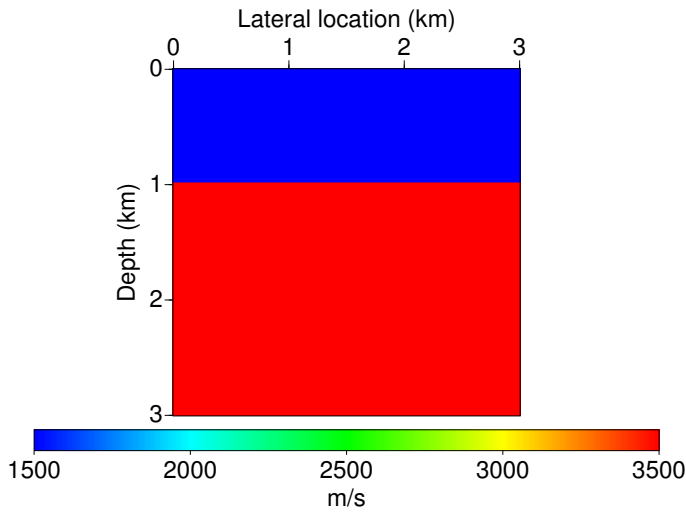

Figura 3: Modelo com refletor na profundidade de 1000m. a) Modelo observado b) Modelo calculado.

(a)

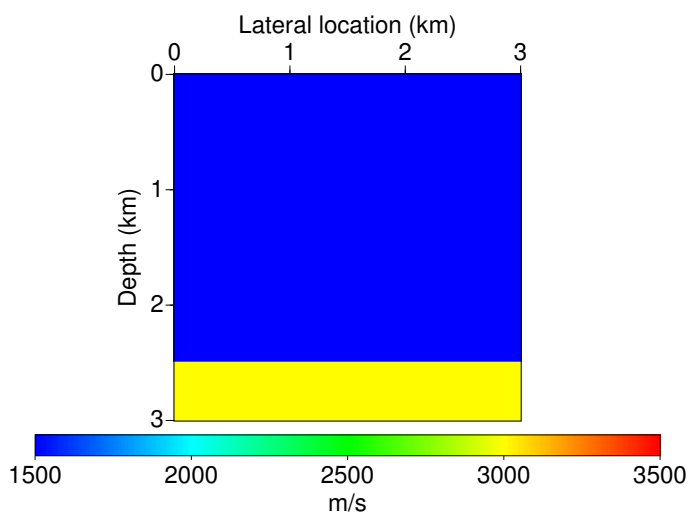

(b)

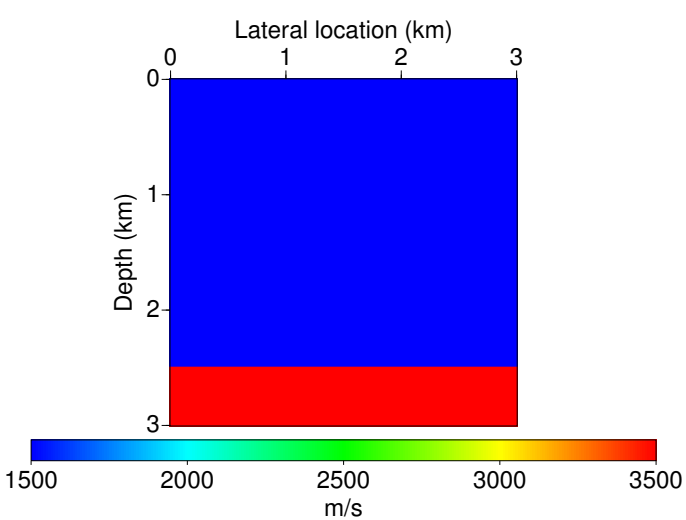

Figura 4: Modelo com refletor na profundidade de $2500 \mathrm{~m}$. a) Modelo observado b) Modelo calculado. (a)

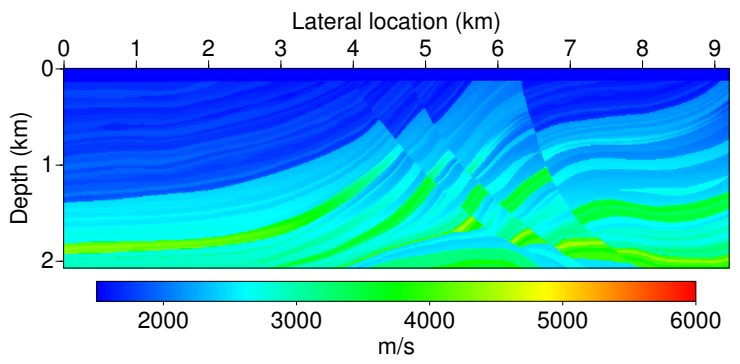

(b)

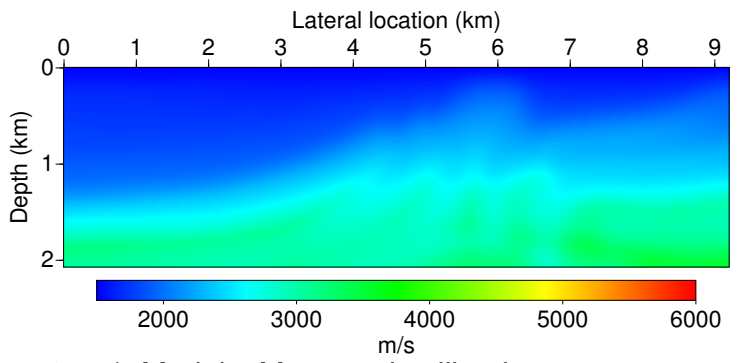

Figura 6: a) Modelo Marmousi utilizado para gerar o dado observado. b) Modelo Marmousi suavizado para ser o modelo inicial.

(a)

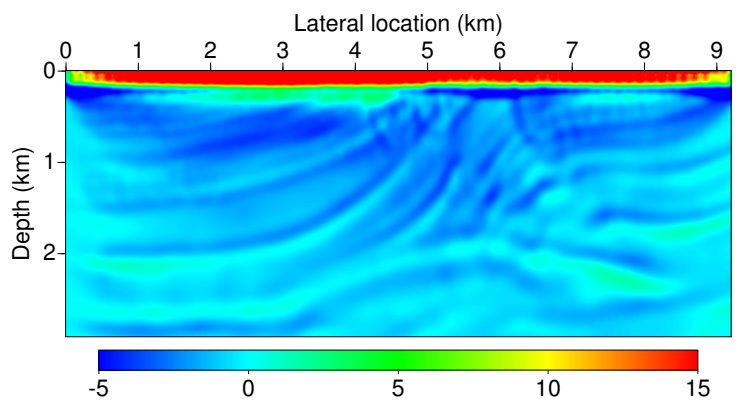

(b)

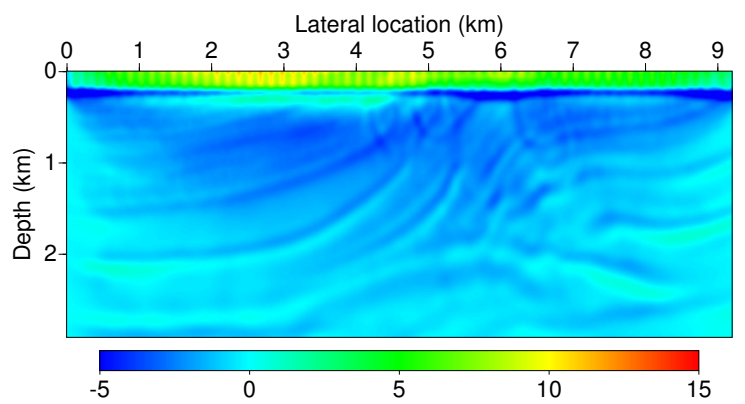

Figura 7: Gradiente para o modelo Marmousi para uma aquisição completa. a) Frequência de $5 \mathrm{~Hz}$. b) Frequência de $10 \mathrm{~Hz}$. 
lâmina de água do modelo. Comparando as imagens, nota-se que o gradiente ficou com uma melhor resolução para a menor frequência.

Um algoritmo que calcula o gradiente da função objetivo para a equação da onda acústica no domínio da frequência foi disponibilizado para que pudesse ser possível a comparação com aqueles produzidos no domínio do tempo. Uma das vantagens do cálculo do gradiente no domínio da frequência é a possibilidade de calculálo para uma única frequência ou para uma banda de frequência, o que faz com que o tempo de processamento do algoritmo seja consideralmente menor do que no domínio do tempo. É mostrada nessa seção a imagem final do gradiente computado no domínio da frequência para diferentes bandas de frequência utilizando o modelo Marmousi mostrado na figura 6. A frequência pico do pulso fonte utilizada nestes testes foi de $5 \mathrm{~Hz}$. A banda de frequência do dado é de cerca de três vezes a frequência pico, ou seja, o algoritmo é capaz de gerar gradientes para uma banda de frequência até cerca de $15 \mathrm{~Hz}$ para este modelo.

\section{Discussão e Conclusões}

Os gradientes para uma única frequência mostram o que esta frequência foi capaz de imagear no gradiente e como ela vai contribuir na recuperação do modelo de velocidade. Nota-se que as imagens para uma única frequência mostram a forma geral do Marmousi enquanto que as imagens do gradiente para uma banda de frequência são mais detalhadas e com mais estruturas aparentes. $\mathrm{O}$ gradiente para a menor banda de frequência $(2 \mathrm{~Hz}$ a $5 \mathrm{~Hz})$ mostrou ter a melhor resolução na imagem, menos distorções são aparentes no fundo do modelo e as estruturas estão bem delimitadas. Observa-se que o aumento da banda de frequência introduz artefatos no gradiente que não estão presentes no modelo de velocidade que se deseja recuperar.

Neste trabalho, os experimentos numéricos para o gradiente no domínio do tempo e no domínio da frequência mostraram que as imagens com melhor distinção de estruturas do modelo foi alcançada com as menores frequências. Para ambos os domínios, o aumento da frequência introduziu artefatos no gradiente que não condiziam com o modelo de velocidade e podem prejudicar a inversão. Além disso, mostrou-se a forma do gradiente em relação a eventos específicos, como o surgimento das banana kernels no gradiente do modelo homogêneo que só produz ondas transmitidas nos campos de onda e o surgimento das rabbit ears kernels no gradiente do modelo com o refletor, que isolou os eventos de reflexão nos campos de onda. Foi possível observar a relação existente entre o offset e a profundidade de iluminação no gradiente, de onde concluiu-se que o máximo de iluminação é atingido em refletores rasos e com longos offsets e apenas os longos offsets foram capazes de iluminar refletores mais profundos. A relação offset vs. frequência também foi analisada e concluiu-se que as baixas frequências e os longos offsets produzem uma resposta melhor no gradiente, o que provavelmente contribuirá para produzir melhores resultados na recuperação do modelo de velocidade. (a)

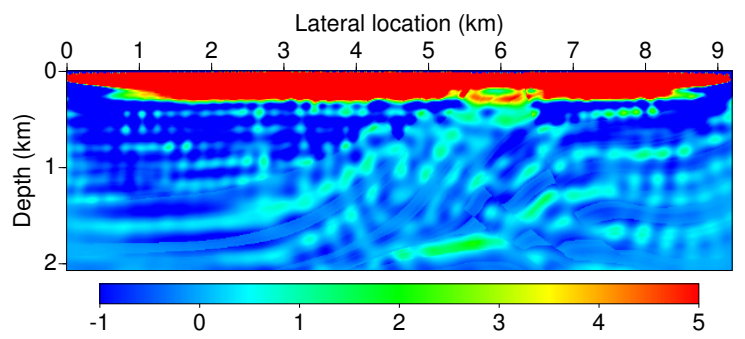

(b)

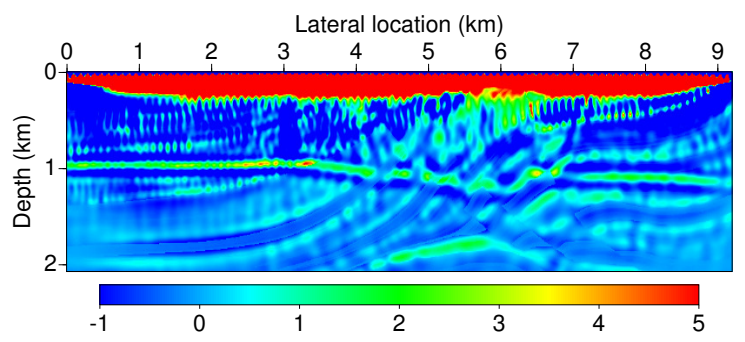

Figura 8: Gradiente para o modelo Marmousi para uma aquisição completa. a) Utilizando uma banda de frequência de $2 \mathrm{~Hz}$ a $5 \mathrm{~Hz}$, com incremento de $0.5 \mathrm{~Hz}$. b) Utilizando uma banda de frequência de $2 \mathrm{~Hz}$ a $12 \mathrm{~Hz}$ com incremento de $1 \mathrm{~Hz}$.

(a)

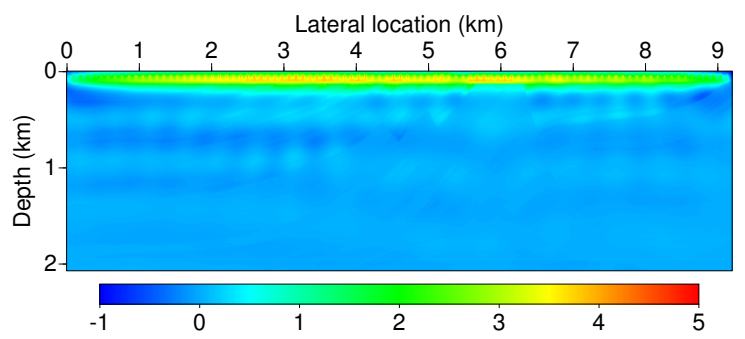

(b)

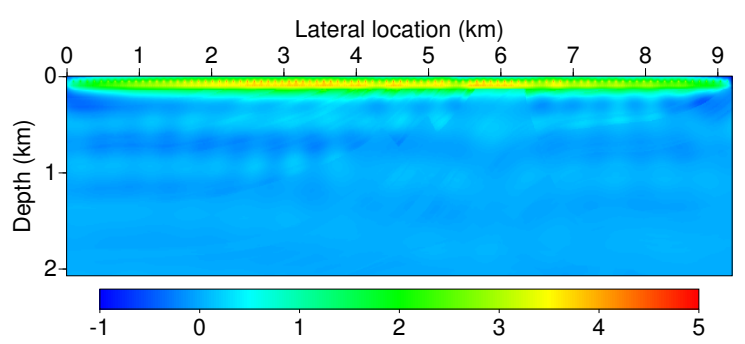

Figura 9: Gradiente para o modelo Marmousi para uma aquisição completa utilizando apenas uma única frequência. a) Apenas para a frequência de $5 \mathrm{~Hz}$. b) Apenas para a frequência de $12 \mathrm{~Hz}$. 


\section{Agradecimentos}

Aos professores Jessé Carvalho Costa e Daniel Leal Macedo pela orientação, ao pós-doutorando Carlos Alexandre Nascimento da Costa pela solicitude e disponibilização dos algoritmos no domínio da frequência e a CAPES, CNPq, CPGf/UFPA e INCT-GP pelo suporte à pesquisa.

\section{Referências}

Claerbout, J., 1971, Toward a unified theory of reflector mapping: Geophysics, 36, 467-481.

Lailly, P., 1983, The seismic inverse problem as a sequence of before stack migrations: Conference on Inverse Scattering, Theory and Application, Society for Industrial and Applied Mathematics, 00, 206-220.

Mora, P. R., 1987, Nonlinear two-dimensional elastic inversion of multi-offset seismic data: Geophysics, 52, 1211-1228.

Pratt, R. G., W. M. H., 1990, Inverse theory applied to multisource cross-hole tomography, Part 1: Acoustic wave-equation method: Geophysical Prospecting, 38, 287-310.

Tarantola, A., 1994, Inversion of seismic reflection data in the acoustic approximation: Canadian Journal of Exploration Geophysics, 49, 1259-1266. 